\title{
Incidence and risk factors for osteoporotic fractures in patients with systemic lupus erythe- matosus versus matched controls
}

Chang Seong Kim ${ }^{1}$, Kyung-Do Han ${ }^{2}$, Jin Hyung Jung ${ }^{2}$, Hong Sang Choi ${ }^{1}$, Eun Hui Bae', Seong Kwon Ma ${ }^{1}$, and Soo Wan Kim ${ }^{1}$

${ }^{1}$ Department of Internal Medicine, Chonnam National University Medical School, Gwangju;

${ }^{2}$ Department of Medical statistics, College of Medicine, The Catholic University of Korea, Seoul, Korea

Received: October 19, 2018 Revised: December 18, 2018 Accepted: February 2, 2019

\section{Correspondence to}

Soo Wan Kim, M.D.

Department of Internal Medicine, Chonnam National University Medical School, 42 Jebong-ro, Dong-gu, Gwangju 61469, Korea Tel: $+82-62-220-6271$

Fax: +82-62-220-8578

E-mail: skimw@chonnam.ac.kr
Background/Aims: Despite recent improvements in the quality of life of patients with systemic lupus erythematosus (SLE), osteoporosis, and osteoporotic fractures are one of the major complications of SLE. Furthermore, limited data are available on the incidence and predictor of osteoporotic fractures in Korean patients with SLE. Herein, we aimed to assess the incidence and risk factors for osteoporotic fractures in Korean SLE patients compared to those without SLE.

Methods: SLE patients aged $\geq 40$ years $(n=10,434$; mean age, $51.3 \pm 9.1$ years; women, $89.7 \%$ ) were selected from the Korean National Health Insurance Service database, spanning a period from 2008 to 2014. Age- and sex-matched controls $(\mathrm{n}=52,170)$ were randomly sampled in a 5:1 ratio from non-SLE individuals. The primary outcome was the first occurrence of osteoporotic fracture.

Results: The incidence of osteoporotic fractures was significantly higher in the SLE patients ( 19.085 per 1,000 person-years) than in matched controls (6.530 per 1,000 person-years). According to the multivariable Cox proportional analysis, patients with SLE exhibited a higher osteoporotic fracture rate than the control group (hazards ratio, 2.964; 95\% confidence interval, 2.754 to 3.188), even after adjustment for confounding variables. In the subgroup analysis, male SLE patients or SLE patients aged 40 to 65 years were associated with a higher osteoporotic fracture rate than women SLE patients or SLE patients aged $\geq 65$ years, respectively. Conclusions: We found a 2.964-fold increased risk of osteoporotic fracture in SLE patients compared to age- and sex-matched non-SLE controls. Male or middle-aged SLE patients had a relatively higher fracture risk among patients with SLE.

Keywords: Osteoporosis; Fracture; Lupus erythematosus, systemic; Risk

\section{INTRODUCTION}

The prevalence of osteoporosis and osteoporotic fractures, which are common metabolic conditions associated with aging, is dramatically increasing due to rapidly aging populations in most developing and developed countries. Since osteoporotic fractures not only reduce quality-of-life but also increase medical and health care expenses, this condition imposes a significant healthcare and financial burden $[1,2]$.

Systemic lupus erythematosus (SLE) is predominant among African Americans, Native Americans, Hispan- 
ics, and Asians, and both the prevalence and incidence of SLE are increasing in the Korean population [3]. In Korea, the prevalence and incidence of SLE between 2006 and 2010 was 20.6 to 26.5 (per 100,000 person) and over 2.5 (per 100,000 person), respectively [4]. SLE is a chronic systemic autoimmune disorder that commonly affects bone homeostasis, which is associated with increased serum levels of various inflammatory cytokines, premature menopause, and maintaining glucocorticoids therapy [5-11]. Recently, a population-based study showed that the incidence ratios for cervical hip fractures in SLE patients were about 3-time higher than those in age- and sex-matched healthy subjects [8]. Moreover, the incidence of fragility fractures in SLE patients increases in younger-aged patients compared to the general population $[6,12]$. However, limited data are available on the incidence, as well as the risk factors, of osteoporotic fractures in Korean patients with SLE.

The aim of the present study was to determine the incidence rate (IR) of osteoporotic fractures between SLE patients and age- and sex-matched controls, as well as to determine the risk factors for osteoporotic fractures among SLE patients, using nationwide population-based data in Korea.

\section{METHODS}

\section{Data source and study population}

We used the national health insurance claims database established by the National Health Insurance Service (NHIS) of Korea [13]. This database includes all claims data provided by the NHIS program and the Medical Aid program. The Korean NHIS program is a compulsory social insurance scheme that covers approximately $97 \%$ of the Korean population; the remaining $3 \%$ are protected under the Medical Aid program [14]. Information on Medical Aid beneficiaries has been integrated into the NHIS database since 2006. Therefore, data extracted from the NHIS database are considered to represent the entire Korean population. The authors obtained approval from the Institutional Review Board of Chonnam National University Hospital, South Korea (CNUH-EXP-2018-026). Informed consent was waived by the Institutional Review Board.

In this study, age, sex, and diagnostic codes based on the International Classification of Diseases, 1oth Revision, Clinical Modification (ICD-10-CM) were retrieved. Patients were categorized as part of the SLE group if they had been treated under M32 of the ICD-10-CM code at least five times between 2008 and 2014; this definition was used previously [15].

Of the 49,884,461 people who underwent at least one health examination between 2008 and 2014, those with a history of fracture during a washout period from 2005 to 2007 to reduce confounding of previous diagnosed fracture or those aged $<40$ years were excluded because osteoporotic fracture is rare in this subpopulation. A total of 10,434 patients with SLE was included in the present study (Supplementary Fig. 1). The subjects comprising the control group were randomly selected from an initial screening of all patients who had undergone an appendectomy or hemorrhoid surgery without a diagnosis of SLE during the same period, such that there were five age- and sex-matched controls per SLE patient.

\section{Definition}

Diabetes was identified based on diagnostic codes (E1O14) from the ICD-10-CM for a self-reported medical history of diabetes or a fasting serum glucose level $\geq 126$ $\mathrm{mg} / \mathrm{dL}$ in the health examination database. Hypertension was defined as previous hypertension diagnosis (I10-13, I15), history of taking at least one antihypertensive drug, or having a blood pressure $\geq 140 / 90 \mathrm{mmHg}$ in the health examination database. Dyslipidemia was identified by the diagnostic code E78, self-reported use of lipid-lowering drugs, or a total cholesterol level $\geq$ $240 \mathrm{mg} / \mathrm{dL}$ in the health examination database. Lower income level was defined at the lowest $20 \%$. Cancer information was collected according to the cancer codes (Coo-96). Stroke and ischemic heart disease were defined by the diagnostic codes I63-64, and I2O-25, respectively. Congestive heart failure was defined by the diagnostic code I5O, and chronic obstructive pulmonary disease (COPD) was identified based on diagnostic codes (J41-J44). End-stage renal disease (ESRD) was defined using the diagnostic codes $\mathrm{N}_{18}-19$ and special medical aid codes for renal replacement therapy (Voo1, Voo3, Voo5). Osteoporotic fracture was defined by diagnostic codes M80 (osteoporosis with pathological fracture), with M81 (osteoporosis without pathological fracture) or M82 (osteoporosis in disease classified as others) being used for 
5 years before these other fracture codes (S22.0, S22.1, $\mathrm{S}_{32.0,} \mathrm{M}_{48.4}$, M48.5, S72.0, $\mathrm{S}_{72.1}, \mathrm{~S}_{42.0}, \mathrm{~S}_{42.2}, \mathrm{~S}_{42.3}, \mathrm{~S}_{52.5}$, S52.6, S82.3, S82.5, and S82.6) [16].

\section{Subgroup analysis}

Subgroup analysis were performed to assess the effect on the risk of osteoporotic fracture in SLE patients, compared with non-SLE individuals, according to sex; age (40 to 64 and > 65 years); degree of income; previous history of either diabetes, hypertension, or dyslipidemia; cancer; COPD; ischemic heart disease; stroke; congestive heart failure, and ESRD.

\section{Statistical analyses}

Prevalence was calculated as the number of cases divided by the total population in 2014. Data were presented as proportions for continuous or categorical variables or mean \pm standard deviation. Multivariable Cox regression analysis was conducted to determine hazard ratio (HR) and the 95\% confidence interval (CI) for associations between SLE and osteoporotic fracture. Calculations were adjusted for age; sex; lower income; and a previous history of either diabetes, hypertension, or dyslipidemia. The incidence probability of osteoporotic fracture according to the presence of SLE was calculated using the Kaplan-Meier method, and the log-rank test was used to analyze differences between study groups. The associations of SLE with osteoporotic fracture were examined in relevant subgroups of patients. Potential interactions were formally tested by including interaction terms. All statistical tests were two-tailed, and $p<$ 0.05 was considered significant. All data analyses were carried out using SAS software version 9.4 (SAS Institute, Cary, NC, USA).

\section{RESULTS}

\section{Characteristics of the study population}

We identified and evaluated the records of 10,434 patients with SLE who were aged at least 40 years as well as 52,170 age- and sex-matched control subjects without SLE for the data analysis. The characteristics of the SLE patients and the age- and sex-matched controls are shown in Table 1. The mean age of the SLE patients and the control group was $51.27 \pm 9.07$ years; those aged over
65 years and women comprised $10.2 \%$ and $89.7 \%$ of the study cohorts, respectively. SLE patients had a significantly higher prevalence of hypertension, diabetes, dyslipidemia, cancer, COPD, ESRD, ischemic heart disease, stroke, and congestive heart failure. There was no difference in income level between both groups.

\section{Increased osteoporotic fracture in SLE patients and matched controls}

The follow-up duration was shorter in SLE patients than in controls $(5.72 \pm 2.43$ years vs. $5.98 \pm 2.30$ years, $p<0.001)$. During a follow-up period, osteoporotic fracture developed more frequently in 1,139 SLE patients (10.9\%) than in 2,036 matched controls $(3.9 \%)(p<0.001)$. Among the patients with osteoporotic fractures, the frequency of osteoporotic fractures was higher in SLE patients than in matched controls before menopause (Table 1). During 59,680 person-years of follow-up, the IR for osteoporotic fracture was 19.085 cases per 1,000 person-years in SLE patients, while IR was 6.530 cases per 1,000 person-years in the control group. SLE patients were independently associated with osteoporotic fracture, even after adjustment for clinical variables (HR, 2.964; 95\% CI, 2.754 to 3.188) (Table 2). Kaplan-Meier curves for the crude 8-year incidence probability of osteoporotic fracture in SLE patients and the control group is shown in Fig. 1. Patients with SLE had significantly higher osteoporotic fracture incidence than non-SLE-matched controls, regardless of gender.

In the subgroup analysis, the pattern of association between SLE and IR for osteoporotic fracture was qualitatively similar across subgroups. Male SLE patients had a higher osteoporotic fracture risk than female SLE patients, even after adjustment for confounding variables ([HR, 4.706; 95\% CI, 3.358 to 6.582] vs. [HR, 2.899; $95 \%$ CI, 2.688 to 3.124], $p$ for interaction $=0.004$ ). Moreover, SLE patients aged between 40 and 65 years had a higher relative risk of osteoporotic fracture than those aged $\geq 65$ years ([HR, 3.383; 95\% CI, 3.102 to 3.687$]$ vs. [HR, 2.223; 95\% CI, 1.927 to 2.556], $p$ for interaction < 0.001 ). SLE patients without a history of cancer, ischemic heart disease, or stroke also had a higher relative risk of osteoporotic fracture than those with a previous history of cancer, ischemic heart disease, or stroke. However, no significant difference was found among SLE patients with respective to income level or a previous history of 
Table 1. Baseline characteristics of the SLE patients and the age- and sex-matched controls

\begin{tabular}{|c|c|c|c|}
\hline \multirow{2}{*}{ Characteristic } & \multicolumn{2}{|c|}{ SLE } & \multirow{2}{*}{$p$ value } \\
\hline & $\mathrm{No}(\mathrm{n}=52,170)$ & Yes $(n=10,434)$ & \\
\hline Female sex & $46,800(89.71)$ & $9,360(89 \cdot 71)$ & 1.000 \\
\hline Age, yr & $51.27 \pm 9.07$ & $51.27 \pm 9.07$ & 1.000 \\
\hline$\geq 65 \mathrm{yr}$ & $5,305(10.17)$ & $1,061(10.17)$ & 1.000 \\
\hline Low income & $13,243(25.38)$ & $2,706(25.93)$ & 0.239 \\
\hline Diabetes mellitus & $3,135(6.01)$ & $728(6.98)$ & 0.001 \\
\hline Hypertension & $9,090(17.42)$ & $3,382(32.41)$ & $<0.001$ \\
\hline Dyslipidemia & $5,404(10.36)$ & $1,754(16.81)$ & $<0.001$ \\
\hline Cancer & $1,493(2.86)$ & $443(4.25)$ & $<0.001$ \\
\hline COPD & $6,490(12.44)$ & $2,622(25.13)$ & $<0.001$ \\
\hline ESRD & $90(0.17)$ & $229(2.19)$ & $<0.001$ \\
\hline Ischemic heart disease & $4,087(7.83)$ & $2,174(20.84)$ & $<0.001$ \\
\hline Stroke & $1,466(2.81)$ & $730(7)$ & $<0.001$ \\
\hline Congestive heart failure & $734(1.41)$ & $510(4.89)$ & $<0.001$ \\
\hline Osteoporotic fracture & $2,036(3.9)$ & $1,139(10.92)$ & $<0.001$ \\
\hline Follow-up duration, yr & $5.98 \pm 2.3$ & $5 \cdot 72 \pm 2.43$ & $<0.001$ \\
\hline Age at osteoporotic facture occurrence, yr & $63.86 \pm 8.95$ & $60.32 \pm 9.44$ & $<0.001$ \\
\hline Frequency of osteoporotic fracture by age group, yr & & & $<0.001$ \\
\hline $40-44$ & $11(0.54)$ & $37(3.25)$ & \\
\hline $45-49$ & $65(3.19)$ & $95(8.34)$ & \\
\hline $50-54$ & $253(12.43)$ & $221(19.4)$ & \\
\hline $55-59$ & $383(18.81)$ & $225(19 \cdot 75)$ & \\
\hline $60-64$ & $359(17.63)$ & $188(16.51)$ & \\
\hline $65-69$ & $385(18.91)$ & $165(14.49)$ & \\
\hline $70-74$ & $321(15 \cdot 77)$ & $112(9.83)$ & \\
\hline$\geq 75$ & $259(12.72)$ & $96(8.43)$ & \\
\hline
\end{tabular}

Values are presented as number (\%) or mean \pm SD.

SLE, systemic lupus erythematosus; COPD, chronic obstructive pulmonary disease; ESRD, end-stage renal disease.

Table 2. Association between SLE and osteoporotic fracture

\begin{tabular}{lrrrrrrr}
\hline SLE & Number & OF & $\begin{array}{c}\text { Follow-up } \\
\text { duration, } \\
\text { person-yr }\end{array}$ & IR $^{\mathrm{a}}$ & ${\text { Model }{ }^{\mathrm{b}}}$ & Model 2 $^{\mathrm{c}}$ & Model $^{\mathrm{d}}$ \\
\hline No & 52,170 & 2,036 & $311,796.75$ & 6.530 & $1(\mathrm{ref})$ & $1(\mathrm{ref})$ & $1(\mathrm{ref})$ \\
Yes & 10,434 & 1,139 & $59,680.29$ & 19.085 & $2.927(2.722-3.146)$ & $3.02(2.808-3.246)$ & $2.964(2.754-3.188)$ \\
\hline
\end{tabular}

Values are presented as hazard ratio (95\% confidence interval).

SLE, systemic lupus erythematosus; OF, osteoporotic fracture; IR, incidence rate.

${ }^{\mathrm{a}}$ IR per 1,000 person-years.

${ }^{\mathrm{b}}$ No adjusted.

${ }^{\mathrm{c}}$ Adjusted for age and sex.

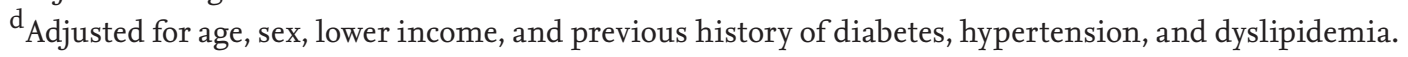



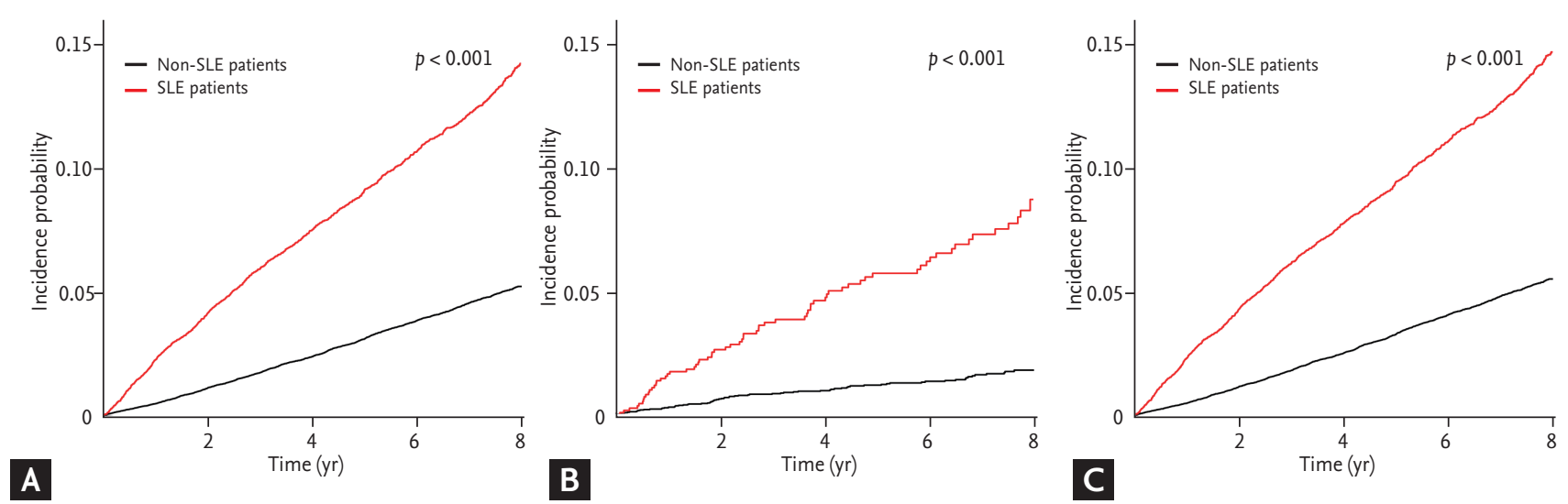

Figure 1. Kaplan-Meier curves for the crude cumulative 8-year osteoporotic fracture between systemic lupus erythematosus (SLE) and non-SLE patients in (A) total, (B) male, and (C) female ( $p$ of log-rank < 0.001 ).

diabetes, hypertension, dyslipidemia, COPD, ESRD, or congestive heart failure (Table 3, Fig. 2).

\section{DISCUSSION}

In this study that analyzed nationwide, population-based data, we found that patients with SLE may have a higher osteoporotic fracture risk than age- and sex-matched control cohorts. There was no significant difference in the osteoporotic fracture risk of SLE patients, with regard to either having a low income level, or a previous history of diabetes, hypertension, dyslipidemia, COPD, ESRD, or congestive heart failure, compared to SLE patients without such characteristics. However, male or younger-aged SLE patients as well as those not having a previous history of either ischemic heart disease, stroke, or cancer showed a higher relative osteoporotic fracture risk among SLE patients.

Although SLE patients have been shown to be at a higher risk of symptomatic vertebral fracture in several small cross-sectional studies [17-20], the association between osteoporotic fracture and SLE in a population-based cohort has not been well characterized. An early retrospective cohort study of 702 female SLE patients found a nearly 5 -fold increase in fracture incidence compared with the general United States female population [7]. In a recent large population-based study from Taiwan, there was an approximately 3.2-fold higher incidence of cervical hip fractures in 14,544 SLE patients than in age- and sex-matched controls, and that fracture risk was elevated starting at a younger age than the general population [8]. Another population-based study in the United Kingdom analyzed a total of 4,343 SLE patients, as well as 21,780 age- and sex-matched controls. This study showed a 1.2-fold increase in the risk of clinical fracture for SLE patients, when compared to controls, using a mean follow-up period of 6.4 years [10]. In line with previous studies, after adjustment for confounding variables, our results showed that SLE patients had a 2.96-fold increase in the risk of osteoporotic fracture, when compared to age- and sex-matched non-SLE controls, which was similar to above study from Taiwan. The reason for the differences in relative risk of fracture between previous population-based studies may be due to racial disparities and disease severities between the different study populations.

Mechanistically, SLE is a chronic inflammatory state that contributes to bone loss by increasing osteoclast activity, while reducing activity of osteoblasts [21]. This appeared to be mediated by the elevated expression of receptor activator of nuclear factor- $\mathrm{kB}$ ligand (RANKL), tumor necrosis factor, and interleukin-1 and $-6[5,22]$. Of note, increased oxidized low-density lipoprotein correlated negatively with low bone-mineral density of the lumbar spine and total hip in SLE patients, which 


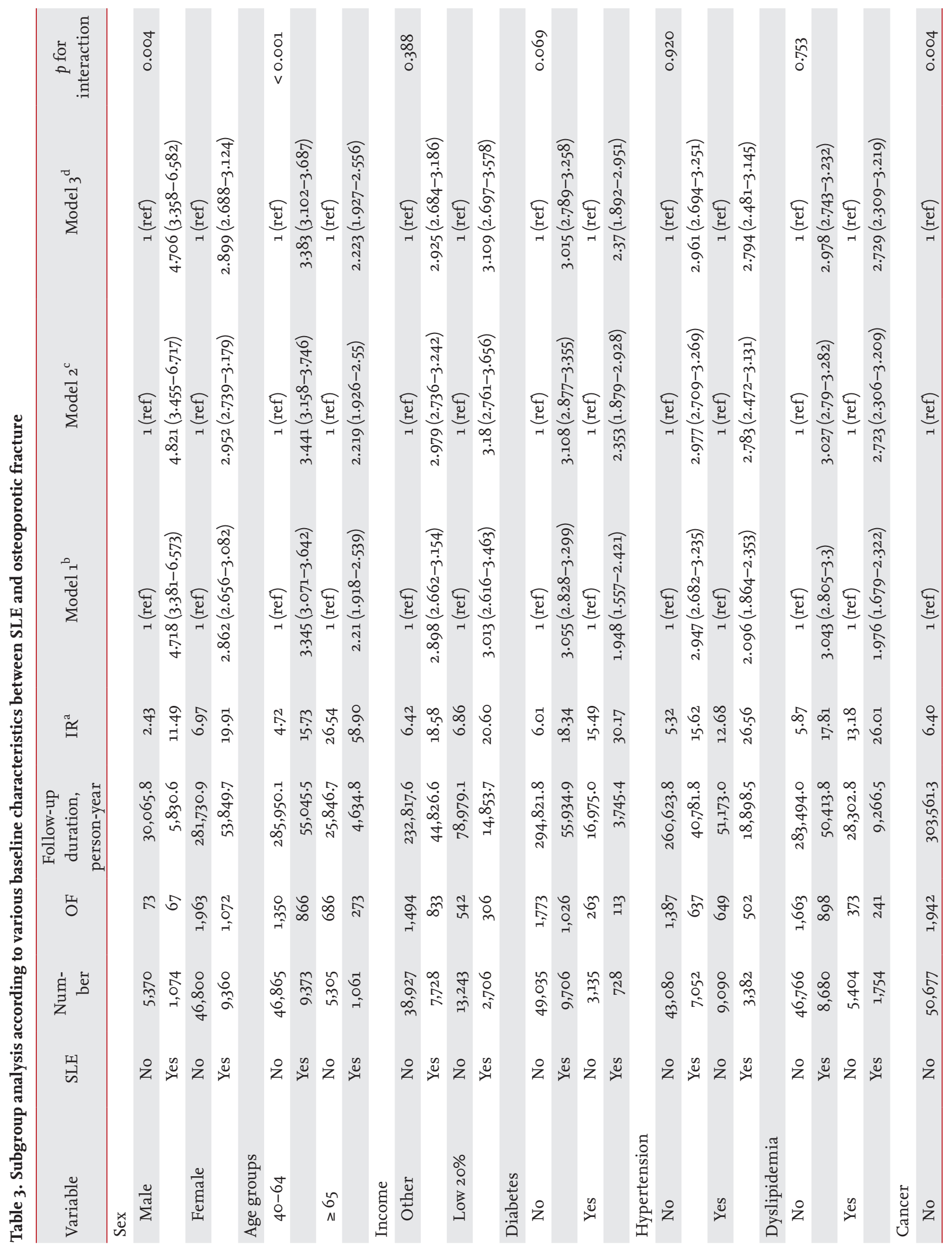




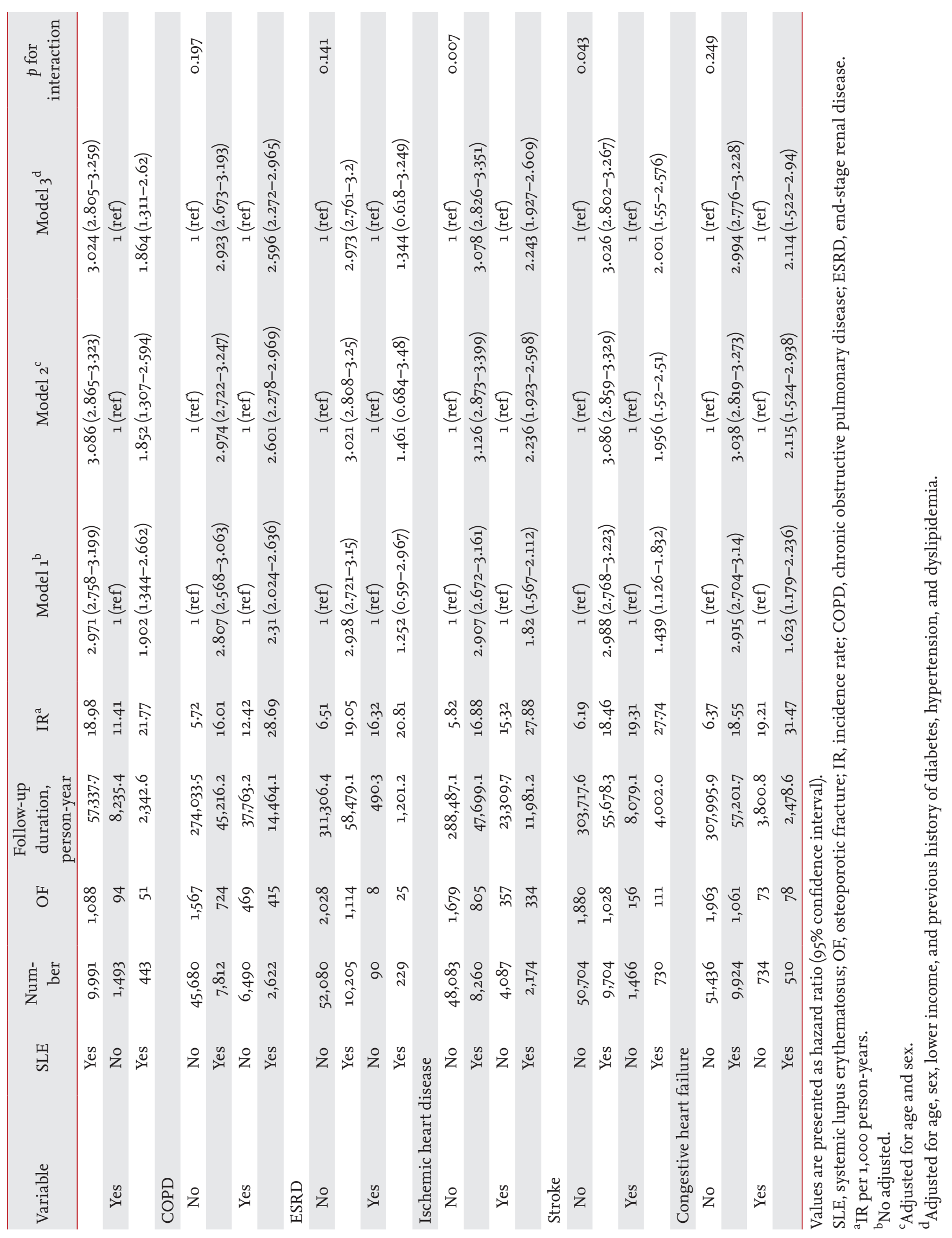




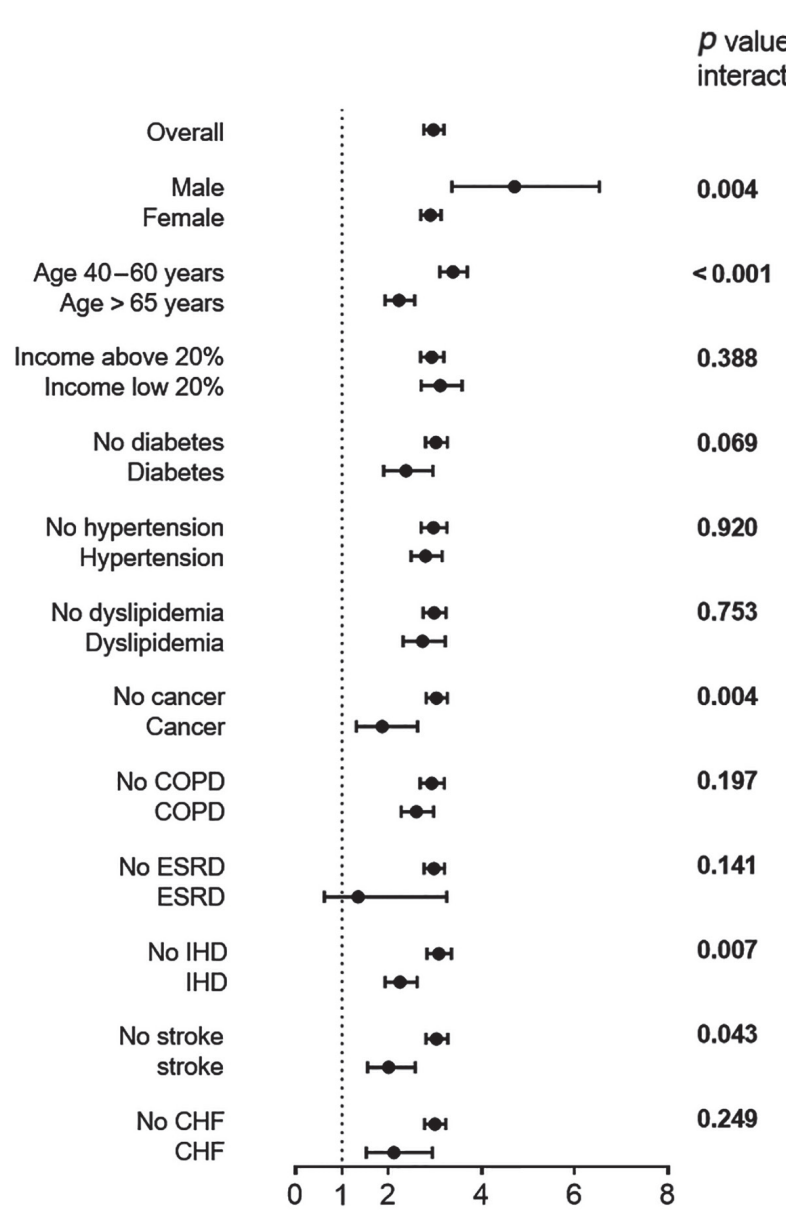

Hazard ratio, $95 \%$ confidence intervals

Figure 2. Overall and subgroup analysis of association between systemic lupus erythematosus (SLE) and osteoporotic fracture in adjusted model. Points and bars represent hazard ratio estimate and $95 \%$ confidence interval, respectively. COPD, chronic obstructive pulmonary disease; ESRD, endstage renal disease; IHD, ischemic heart disease; CHF, congestive heart failure.

may attenuate osteoblast maturation and increase the expression of RANKL, which may eventually induce osteoporosis [23]. Moreover, SLE patients may have lower vitamin D levels as a result of the avoidance of sun exposure, decreased renal function, and the use of glucocorticoids, anticonvulsants, hydroxychloroquine, and calcineurin inhibitors. Low levels of vitamin $\mathrm{D}$ is also associated with disease severity via inappropriate immune response and has been shown to be correlated with osteoporosis in SLE patients [21,24].

Notwithstanding the fact that men comprised $10 \%$ of the study cohort, which is similar to previous SLE co- hort studies $[8,10,25]$, our results identified male SLE patients have a high risk of osteoporotic fracture in the SLE cohort according to subgroup analysis. Similar to our findings, a study from United Kingdom showed a higher relative risk of clinical fracture in male SLE patients than in female SLE patients (adjusted relative risk, 1.91 vs. 1.18), although statistical significance was not found [10]. Furthermore, SLE patients aged 40 to 65 years were at an increased relative risk of osteoporotic fracture when compared to SLE patients older than 65 years. This finding was consistent with a previous study from Taiwan that found the incidence of hip fracture was higher in SLE patients younger than 50 years than in those aged $\geq 50$ years [8]. Although the IR of osteoporotic fracture increases with age among the SLE cohort (aged 40 to 65 years, 7.4 cases per 1,000 person-years; aged $\geq$ 65 years, 32.6 cases per 1,000 person-years), the relative risk was higher for younger SLE patients than for older SLE patients when compared with matched controls. In other words, despite the incidence of osteoporotic fracture is low in male and young general population, male or younger SLE patients had a relatively higher fracture risk than female or older SLE patients, which suggested that SLE is associated with the risk of osteoporotic fracture. One possible explanation is that SLE patients have a higher rate of premature menopause and the use of glucocorticoid at a younger age.

The findings of the present study provide the first evidence of an independent association between SLE and the risk of osteoporotic fracture using a larger, nationwide population-based cohort from Korea. Consequently, we believe that our findings could be used as a fundamental data for treatment and future prevention of osteoporotic fracture in Korean SLE patients by understanding of risk factors for osteoporotic fracture. However, there are several limitations to this study. First, our data did not identify the use of drugs such as glucocorticoids, anticonvulsants, hydroxychloroquine, calcineurin inhibitors, vitamin D supplements, and bisphosphonates, which could affect the risk of osteoporotic fracture. Second, we did not evaluate the association of osteoporotic fracture risk with SLE patients according to other sites, such as the vertebrae, hip, ankle, or wrist. Future studies are required to determine the influence of drug use and fracture site on the risk of osteoporotic fractures. 
In this large population-based cohort study, we found that there was an approximately 3-fold increased risk of osteoporotic fracture in SLE patients, when compared to age- and sex-matched non-SLE controls. Male or middle-aged SLE patients had a relatively higher fracture risk among SLE patients. Therefore, strategies to prevent osteoporotic fracture, including the treatment of osteoporosis in SLE patients, should be examined in the future, especially in both male and younger patients.

\section{KEY MESSAGE}

1. We found a 2.96-fold increased risk of osteoporotic fracture in systemic lupus erythematosus (SLE) patients compared to age- and sexmatched non-SLE controls.

2. Male or middle-aged SLE patients had a relatively higher fracture risk among patients with SLE.

\section{Conflict of interest}

No potential conflict of interest relevant to this article was reported.

\section{Acknowledgments}

This research was supported by Basic Science Research Program through the National Research Foundation (NRF) of Korea funded by the Ministry of Science and ICT (MSIT) (NRF-2015R1C1A1A01051769), by the Bio \& Medical Development Program through the NRF of Korea funded by the MSIT (NRF-2017M3A9E8023018), and by the Basic Science Research Program through the NRF of Korea funded by the Ministry of Education (NRF-2018R1D1A1Bo7042999).

\section{REFERENCES}

1. Kim JW, Jeon YJ, Baek DH, Kim TN, Chang JS. Percentage of the population at high risk of osteoporotic fracture in South Korea: analysis of the 2010 fifth Korean National Health and Nutrition Examination Survey data. Osteoporos Int 2014;25:1313-1319.

2. Burge R, Dawson-Hughes B, Solomon DH, Wong JB, King A, Tosteson A. Incidence and economic burden of osteoporosis-related fractures in the United States, 20052025. J Bone Miner Res 2007;22:465-475.

3. Jakes RW, Bae SC, Louthrenoo W, Mok CC, Navarra SV, Kwon N. Systematic review of the epidemiology of systemic lupus erythematosus in the Asia-Pacific region: prevalence, incidence, clinical features, and mortality. Arthritis Care Res (Hoboken) 2012;64:159-168.

4. Shim JS, Sung YK, Joo YB, Lee HS, Bae SC. Prevalence and incidence of systemic lupus erythematosus in South Korea. Rheumatol Int 2014;34:909-917.

5. Tang XL, Griffith JF, Qin L, et al. SLE disease per se contributes to deterioration in bone mineral density, microstructure and bone strength. Lupus 2013;22:1162-1168.

6. Yee CS, Crabtree N, Skan J, et al. Prevalence and predictors of fragility fractures in systemic lupus erythematosus. Ann Rheum Dis 2005;64:111-113.

7. Ramsey-Goldman R, Dunn JE, Huang CF, et al. Frequency of fractures in women with systemic lupus erythematosus: comparison with United States population data. Arthritis Rheum 1999;42:882-890.

8. Wang SH, Chang YS, Liu CJ, et al. Association of systemic lupus erythematosus with a higher risk of cervical but not trochanteric hip fracture: a nationwide population-based study. Arthritis Care Res (Hoboken) 2013;65:1674-1681.

9. Lee C, Almagor O, Dunlop DD, Manzi S, Spies S, Ramsey-Goldman R. Self-reported fractures and associated factors in women with systemic lupus erythematosus. J Rheumatol 2007;34:2018-2023.

10. Bultink IE, Harvey NC, Lalmohamed A, et al. Elevated risk of clinical fractures and associated risk factors in patients with systemic lupus erythematosus versus matched controls: a population-based study in the United Kingdom. Osteoporos Int 2014;25:1275-1283.

11. Bultink IE, Lems WF. Systemic lupus erythematosus and fractures. RMD Open 2015;1:e000069.

12. Varacallo MA, Fox EJ. Osteoporosis and its complications. Med Clin North Am 2014;98:817-831.

13. Choi JB, Lee EJ, Han KD, Hong SH, Ha US. Estimating the impact of body mass index on bladder cancer risk: stratification by smoking status. Sci Rep 2018;8:947.

14. Lee SR, Choi EK, Han KD, Cha MJ, Oh S. Trends in the incidence and prevalence of atrial fibrillation and estimated thromboembolic risk using the $\mathrm{CHA}(2) \mathrm{DS}(2)$ VASc score in the entire Korean population. Int J Cardiol 2017;236:226-231.

15. Yun JS, Bae JM, Kim KJ, et al. Increased risk of thyroid 
diseases in patients with systemic lupus erythematosus: a nationwide population-based study in Korea. PLoS One 2017;12:e0179088.

16. Kim J, Lee E, Kim S, Lee TJ. Economic burden of osteoporotic fracture of the elderly in South Korea: a national survey. Value Health Reg Issues 2016;9:36-41.

17. Bultink IE, Lems WF, Kostense PJ, Dijkmans BA, Voskuyl AE. Prevalence of and risk factors for low bone mineral density and vertebral fractures in patients with systemic lupus erythematosus. Arthritis Rheum 2005;52:2044-2050.

18. Mendoza-Pinto C, Garcia-Carrasco M, Sandoval-Cruz $\mathrm{H}$, et al. Risk factors of vertebral fractures in women with systemic lupus erythematosus. Clin Rheumatol 2009;28:579-585.

19. Borba VZ, Matos PG, da Silva Viana PR, Fernandes A, Sato EI, Lazaretti-Castro M. High prevalence of vertebral deformity in premenopausal systemic lupus erythematosus patients. Lupus 2005;14:529-533.

20. Li EK, Tam LS, Griffith JF, et al. High prevalence of as- ymptomatic vertebral fractures in Chinese women with systemic lupus erythematosus. J Rheumatol 2009;36:16461652.

21. Edens C, Robinson AB. Systemic lupus erythematosus, bone health, and osteoporosis. Curr Opin Endocrinol Diabetes Obes 2015;22:422-431.

22. Lacativa PG, Farias ML. Osteoporosis and inflammation. Arq Bras Endocrinol Metabol 2010;54:123-132.

23. Sun YN, Feng XY, He L, et al. Prevalence and possible risk factors of low bone mineral density in untreated female patients with systemic lupus erythematosus. Biomed Res Int 2015;2015:510514.

24. Mok CC. Vitamin D and systemic lupus erythematosus: an update. Expert Rev Clin Immunol 2013;9:453-463.

25. Ekblom-Kullberg S, Kautiainen H, Alha P, Leirisalo-Repo $\mathrm{M}$, Julkunen $\mathrm{H}$. Frequency of and risk factors for symptomatic bone fractures in patients with systemic lupus erythematosus. Scand J Rheumatol 2013;42:390-393. 


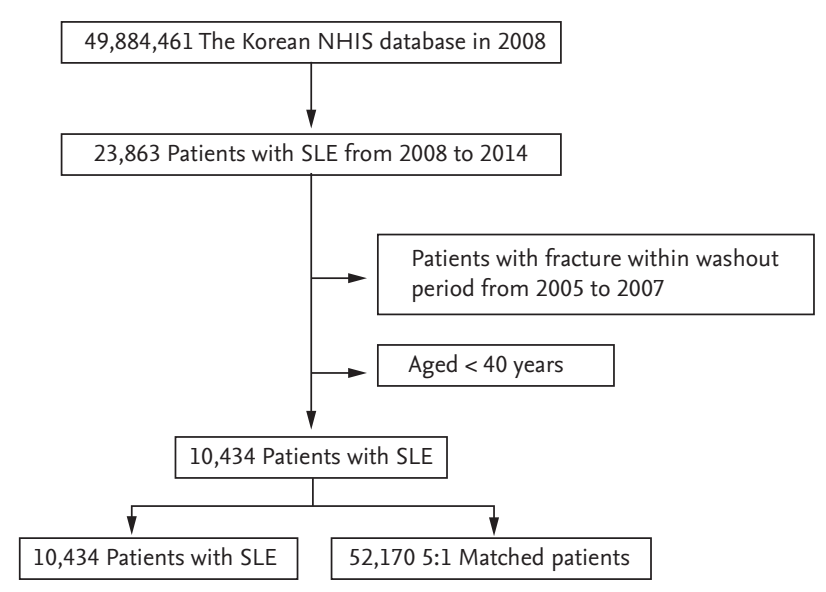

Supplementary Figure 1. Flow chart of the study. NHIS, National Health Insurance Service; SLE, systemic lupus erythematosus. 